2010-06

\title{
Temporal Distance and Person Memory: Thinking About the Future Changes Memory for the Past
}

Wyer, Natalie

http://hdl.handle.net/10026.1/2943

\subsection{7/0146167210370965}

Personality and Social Psychology Bulletin

SAGE Publications

All content in PEARL is protected by copyright law. Author manuscripts are made available in accordance with publisher policies. Please cite only the published version using the details provided on the item record or document. In the absence of an open licence (e.g. Creative Commons), permissions for further reuse of content should be sought from the publisher or author. 


\section{Personality and Social Psychology Bulletin \\ http://psp.sagepub.com/}

Temporal Distance and Person Memory: Thinking About the Future Changes Memory for the Past

Natalie A. Wyer, Timothy J. Perfect and Sabine Pahl

Pers Soc Psychol Bull 2010 36: 805 originally published online 5 May 2010

DOI: $10.1177 / 0146167210370965$

The online version of this article can be found at:

http://psp.sagepub.com/content/36/6/805

Published by:

(3)SAGE

http://www.sagepublications.com

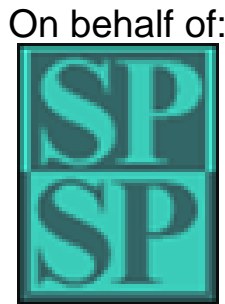

Society for Personality and Social Psychology

Additional services and information for Personality and Social Psychology Bulletin can be found at:

Email Alerts: http://psp.sagepub.com/cgi/alerts

Subscriptions: http://psp.sagepub.com/subscriptions

Reprints: http://www.sagepub.com/journalsReprints.nav

Permissions: http://www.sagepub.com/journalsPermissions.nav

Citations: http://psp.sagepub.com/content/36/6/805.refs.html 


\section{Temporal Distance and Person Memory: Thinking About the Future Changes Memory for the Past}

Personality and Social

Psychology Bulletin

36(6) 805-816

(C) 2010 by the Society for Personality

and Social Psychology, Inc

Reprints and permission:

sagepub.com/journalsPermissions.nav

DOI: $10.1177 / 0146167210370965$

http://pspb.sagepub.com

@SAGE

\author{
Natalie A. Wyer', Timothy J. Perfect', and Sabine Pahl'
}

\begin{abstract}
Psychological distance has been shown to influence how people construe an event such that greater distance produces highlevel construal (characterized by global or holistic processing) and lesser distance produces low-level construal (characterized by detailed or feature-based processing). The present research tested the hypothesis that construal level has carryover effects on how information about an event is retrieved from memory. Two experiments manipulated temporal distance and found that greater distance (high-level construal) improves face recognition and increases retrieval of the abstract features of an event, whereas lesser distance (low-level construal) impairs face recognition and increases retrieval of the concrete details of an event. The findings have implications for transfer-inappropriate processing accounts of face recognition and event memory, and suggest potential applications in forensic settings.
\end{abstract}

\title{
Keywords
}

psychological distance, person memory, construal level

Received February 6, 2009; revision accepted November 15, 2009

On a daily basis, we encounter many people and witness many events. Most of the time, we do not give these people and events a second thought. However, there are occasions when we need to recognize a person we have seen earlier or to reconstruct a sequence of events we have witnessed. The ease and accuracy with which we are able to do so depend on a number of factors. One such factor is the processing style that we employ-either global processing, in which we attend to the general meaning of an event and the relationships among its elements, or detailed processing, in which we attend primarily to the elements themselves. The present research conceptualizes processing style within the framework of construal-level theory, testing the effect of temporal distance on person and event memory in naturalistic social interactions.

When it comes to recognizing faces, evidence suggests that a global processing style optimizes our ability to accurately identify a person we have encountered earlier. This is because the act of perceiving a face involves more than picking out individual facial features. Each face is characterized by a unique configuration of those features, and that configural information is critical to the ability to recognize the face. Global processing encourages attention to configural information (Macrae \& Lewis, 2002) and thus is best suited to the task of recognizing faces. Indeed, when perceivers are unable to use configural information (e.g., when a face is inverted or when the top and bottom of different faces are combined in a composite face; see Yin, 1969; Young, Hellawell, \& Hay, 1987), recognition performance suffers.

Previous research indicates that manipulations that encourage perceivers to shift away from global processing toward a more detailed (i.e., feature-based) processing style disrupt face-recognition processes. For example, studies have shown that a period of verbal description before a lineup of faces results in poorer recognition performance, an effect called verbal overshadowing (Schooler \& Engstler-Schooler, 1990). This effect occurs whether the verbal description is of the target person, a different person, or even an object (Dodson, Johnson, \& Schooler, 1997; Westerman \& Larsen, 1997). This effect has been explained as a form of transfer-inappropriate processing (Schooler, 2002) whereby the task of description is thought to encourage participants to focus on detailed or featural information that can be easily verbalized. This focus carries over to the recognition task, where it interferes with

\footnotetext{
'University of Plymouth, Plymouth, United Kingdom

Corresponding Author:

Natalie A.Wyer, School of Psychology, University of Plymouth, Drake

Circus, Plymouth PL4 8AA, United Kingdom

Email: natalie.wyer@plymouth.ac.uk
} 
the global processing that would normally help identification of the target.

Further evidence for the transfer-inappropriate processing account derives from research by Macrae and Lewis (2002). In their experiment, participants were first exposed to a target person before being presented with a series of Navon stimuli (i.e., global letters composed of smaller constituent letters) and were asked to report either the elements of stimuli (in the local condition) or the overall shape of the stimuli (in the global condition). Participants were later given a recognition task in which they were asked to identify from a lineup the person they had seen earlier. Macrae and Lewis discovered that participants in the global condition were significantly more likely to correctly identify the target compared to those in a no-Navon baseline condition. In contrast, those in the local condition were significantly less likely to do so. Macrae and Lewis concluded that global instructions for performing the Navon task enhanced participants' use of global processing, thereby enhancing face recognition, whereas local instructions shifted participants toward detailed processing, which disrupted face recognition.

Macrae and Lewis's (2002) effects have been replicated both in the laboratory (Perfect, 2003; Weston, Perfect, Schooler, \& Dennis, 2008), and in field studies (Perfect, Dennis, \& Snell, 2007). Weston and Perfect (2005) also demonstrated the complementary effect whereby orienting to the local features of the Navon letters speeded subsequent performance on a feature-based face-recognition task, namely, identification of half-faces in a composite-face test. These studies collectively provide compelling evidence for a processing bias effect on face recognition.

Thus, the transfer-inappropriate processing account has received substantial support from studies in which participants are explicitly required to focus on global or local details of a stimulus and where the final test is recognition based. However, previous research has focused on perceptual processes by manipulating attention to spatial features or configurations, or investigating the way in which verbalization interferes with those processes. In the present work, we apply construal-level theory to person memory for the first time, exploring the effects of temporal distance on subsequent face-recognition memory in Experiment 1, before extending this to alternate forms of person memory in Experiment 2. To the extent that manipulations of temporal distance (and resulting shifts in construal level) have an effect on person memory, this would suggest that face processing is influenced not only by manipulations that are closely linked with face perception but also by broader differences in global versus detailed processing style.

Recent research in social cognition suggests that global and local processing can be elicited by simply shifting perceivers' temporal distance from an event. When asked to consider an event in the near future or recent past, people tend to focus on the details (what exactly happened, in what order).
In contrast, when asked to think about an event in the distant future or distant past, they are more likely to perceive it abstract terms (why the event occurred, what it meant). Trope and Liberman (2003; see also Liberman \& Trope, 2008; Liberman, Trope, \& Stephan, 2007; Trope, Liberman, \& Wakslak, 2007) have argued that such findings are evidence that psychological distance has direct consequences for construal level. Psychologically close events are construed at a low level (i.e., in terms of their specific details) whereas psychologically distant events are construed at a high level (i.e., in terms of their broader meaning).

Evidence has now accumulated that temporal distance influences a wide variety of judgments, and it seems to do so by shifting people toward either a high-level global processing style or a low-level detailed processing style. In light of the previously discussed literature on transfer-inappropriate processing as applied to face recognition, this raises the intriguing question of whether the same effects might be brought about by manipulations of temporal distance. Indeed, Liberman and Förster (2009) have recently suggested that psychological distance may influence face recognition. Recent work by Hunt and Carroll (2008) supports this proposal, and it provides the starting point for the present line of research. In Hunt and Carroll's study, participants initially studied a photograph of a face for 10 seconds under explicit study instructions. Following a 5-minute delay, half the participants generated a description of the face they had seen, and half completed a filler task, as in the standard verbal overshadowing paradigm. Participants were then randomly assigned to a control condition, a near-future condition in which they imagined their next day, or a distant-future condition in which they imagined a day in their life in 1 year's time. Participants then completed two performance measures. The first was a set of insight problem-solving tasks for 2 minutes, and the second was a lineup featuring the face they had seen earlier alongside five similar foil faces.

Consistent with the verbal overshadowing literature, Hunt and Carroll (2008) found that a verbal description impaired subsequent recognition performance. However, they also reported that this decrement was overcome by orienting people toward the distant future. In contrast, orientation toward the near future left the verbal overshadowing effect unaltered. The authors argued that invoking greater psychological distance reinstated a high-level (i.e., global) processing style and thus returned perceivers to normal performance levels. They also reported that temporal focus influenced insight problem solving (believed to be associated with global processing), with those in the distant-future condition solving more problems than those in the near-future or control conditions, thus providing an independent measure of processing bias.

Although these findings are suggestive, they also raise a number of questions about the impact of temporal focus on recognition memory. One such question involves the 
unexpected absence of a temporal focus effect in the nodescription control condition. If, as the authors argued, temporal focus has the effect of shifting processing style, then one would have expected to find better recognition performance in the no-description control group following distantfuture focus, and worse performance following near-future focus, in line with the effects of Navon orientation reported by Macrae and Lewis (2002). Neither effect was apparent. The results of the insight problem-solving task are also only suggestive. Because performance on this task is only reported as a function of temporal distance (collapsing across the description and no-description control conditions), conclusions regarding the extent to which insight task performance (i.e., global processing) was related to face recognition are obscured. Moreover, the reported pattern shows a dissociation from that found in the recognition data. The insight task revealed that the distant-future condition showed superior performance to the other two conditions, which did not differ. However, the recognition data, collapsed across description and no-description control in the same manner, showed that it was the near-future condition that differed from the distant-future and control conditions, which did not differ from each other (correct identification rates: near-future focus, $50 \%$; distant-future focus, $72.1 \%$; control, $71.3 \%$ ). Thus, it is not clear that the recognition task and insight problemsolving tasks are both influenced by temporal distance in the same manner. The use of insight problem solving as a measure of high-level construal is also problematic because the engagement with these tasks might have carryover effects on the final recognition task that followed.

The difficulty in interpreting Hunt and Carroll's (2008) findings stem in part from the complexity of the design, in which participants first described (or not) a face and then were subject to the manipulation of temporal focus. Additionally, participants completed a number of other tasks of unknown construal level (arithmetic tasks, spot-the-difference tasks, a puzzle task, and insight problem-solving tasks were all used at different phases of the study). The authors' interpretation of the interaction observed between verbal description and temporal focus rests on the assumption that the effects are additive and that engagement in the other tasks had no effect. However, given the absence of a construal effect in the no-description control condition, these assumptions are open to challenge. Another problem with the study, from a theoretical perspective, is that participants were also allowed to opt out of selecting a face from the lineup. Although this provides ecological validity (as real witnesses may reject a lineup entirely), it complicates any interpretation of the recognition data because the construal manipulation could have influenced response bias rather than accuracy.

Thus, in the present research, we investigated the effects of temporal distance in a simpler design. To maintain the ecological focus of our earlier Navon-based research (Perfect et al., 2007) we used a naturalistic encounter between the participant and a confederate as the target memory. Our earlier research has shown that this method produces reliable Navon effects, replicating those reported by Macrae and Lewis (2002). As well as being closer to the realistic conditions under which a witness may encounter a suspect, this method has the methodological advantage of preventing participants from adopting deliberate encoding strategies at study or from rehearsing the face during the retention interval because the final memory test for the confederate is not introduced until after the construal manipulation has been induced. Consequently, any effects of construal level cannot be attributed to such encoding or rehearsal strategies.

Our use of a real-life encounter as the target event represents a first attempt to study the effects of temporal distance (and resulting construal level) in a natural setting. Although manipulations of psychological distance have proven to be powerful in laboratory experiments, to our knowledge they have not be applied in the field, and thus the extent to which they are robust in more complex environments remains to be seen. Indeed, although incidental social encounters are relatively common in research on eyewitness memory, research on person memory has tended to focus on laboratory-based paradigms that offer greater experimental control. In recognition of this, a further aim of our research is to explore the effects of psychological distance on person memory in the context of a relatively complex face-to-face encounter.

\section{Overview of Experiments}

In Experiment 1, we address the question of whether psychological distance influences both face-recognition performance and processing style. According to construal-level theory, psychological distance should induce global processing whereas psychological proximity should induce detailed (feature-based) processing, which should also influence face-recognition accuracy. In Experiment 2, we replicate and extend the first experiment by exploring other aspects of person memory, such as memory for behavioral and trait information, which processing style (and hence construal level) might be expected to influence. Traditional investigations of person perception (e.g., Hamilton, Katz, \& Leirer, 1980; Wyer, Bodenhausen, \& Srull, 1984) have demonstrated that one's memory for another individual is likely to include both abstract information (e.g., personality traits that the person is believed to possess) and more concrete information (e.g., specific instances in which the person behaved in a particular way). To the extent that construal level influences processing of stored person representations, it might be expected to shape the nature of information that is retrieved from that representation. For example, global processing (resulting from high-level construal) might result in retrieval of relatively abstract trait information whereas detailed processing (resulting from low-level construal) might result in retrieval of relatively concrete behavioral information. Taken together, 
the present research provides a novel application of construallevel theory to person and event memory, using a naturalistic incidental social interaction.

\section{Experiment I}

The aims of Experiment 1 are twofold. First, we seek to replicate the findings reported by Macrae and Lewis (2002) using a manipulation of processing style that does not require participants to process spatial information in a way that parallels face processing. Macrae and Lewis's use of Navon stimuli to impose global versus detailed processing drew participants' attention to elemental or configural information in a manner that is superficially similar to strategies known to disrupt or improve face processing. However, if processing style (and not attention to certain aspects of spatial information) underlies the effects they report, then manipulations that do not involve spatial information should produce parallel results. To test this idea, we adopted the temporal-focus manipulation used by Hunt and Carroll (2008). A second goal of Experiment 1 was to obtain an independent indicator of how construal influences processing style in the primary (manipulation) task. The majority of previous research on processing style and face recognition has relied on inferring differences in processing from differences in recognition accuracy. The one exception is the study by Hunt and Carroll, who measured processing style by means of insight problem solving. However, as we argued previously, interpreting their results is complicated by a number of factors. Consequently, Experiment 1 will provide a direct test of the hypothesis that temporal distance influences processing style by means of a questionnaire asking participants to indicate which features of the future event they thought about. We expect that thinking about the distant future will lead to more reports of thinking about the meaning of the event (high construal), which will be associated with better face recognition on the final test. Conversely, thinking about the near future will lead to higher reports of thinking about the detail of the event (low construal), and poorer face-recognition performance.

\section{Method}

Participants. Ninety volunteers (45 male, 45 female) were approached on campus and agreed to participate in the present study. Their ages ranged from 18 to 51 years, with a mean of $23.4(S D=7.39)$. No other biographical data were collected.

Procedure. Participants were approached on campus by a male confederate who claimed to be lost and looking for a particular location. The confederate engaged each participant in conversation for up to 2 minutes before exiting in the direction indicated by the participant. Once the confederate was out of view, the experimenter approached the same individual and asked them to participate in a brief psychology study about being at the university.

Those in the two experimental conditions were asked to imagine that they had to give a presentation to prospective students visiting the university at an Open Day. It was made clear to participants that this was a purely hypothetical scenario. Participants in the distant-future condition $(n=30)$ were told to imagine that the presentation would take place after the summer examination period (approximately 5-7 months later) in an unspecified location on campus. Those in the near-future condition $(n=30)$ were told to imagine that the presentation would take place at 3:00 p.m. the next day, and they were given the name of a large lecture theater on campus. Participants were given 2 minutes to think about this scenario before completing a 10-item questionnaire that asked them what aspects of the scenario they thought about. Half the items in the questionnaire aimed at testing specific details of the event (e.g., "I thought about how many slides I would use," "I thought about how long the presentation would last"), with the other half designed to test global aspects of the event (e.g., "I thought that it would be good for my career," "I thought about how it would benefit the university"). Each questionnaire item was presented as a statement, along with a 5-point Likert-type scale ranging from 1 (strongly disagree) to 5 (strongly agree).

Those in the control condition $(n=30)$ were asked to complete a brief 10 -question survey about their attitudes toward life as a student. Participants were asked to think about the questions for 2 minutes before completing the questionnaire. Each question consisted of a brief statement about student life (e.g., "I have enjoyed my time at University," "I'm glad I chose the course I did"), along with a 5-point Likerttype scale ranging from 1 (strongly disagree) to 5 (strongly agree). This questionnaire was used solely as a filler task to occupy the participants for the same duration as those in the experimental conditions, and it was not analyzed further.

Upon completion of the questionnaire, participants in all conditions were informed that the earlier interaction with the confederate had been part of the study and that, if they agreed, they would be asked to attempt to identify the confederate from a lineup. All participants agreed to participate. They were then shown an eight-person simultaneous targetpresent photographic lineup and asked to identify the confederate they had interacted with previously. There was no "not in lineup" option. The photographs were color, each sized $55 \times 70 \mathrm{~mm}$, depicting males with dark hair, aged 18-30 years, in a full-frontal head and shoulders pose. No attempt was made to hide clothing, but the target was depicted wearing different clothes from those seen during the encounter. The photographs were presented in a $2 \times 4$ matrix on a single A4 sheet, labeled A to D (top row) and E to $\mathrm{H}$ (bottom row), with the target in position F. Participants were allowed to take as long as they wished to make their verbal response. 


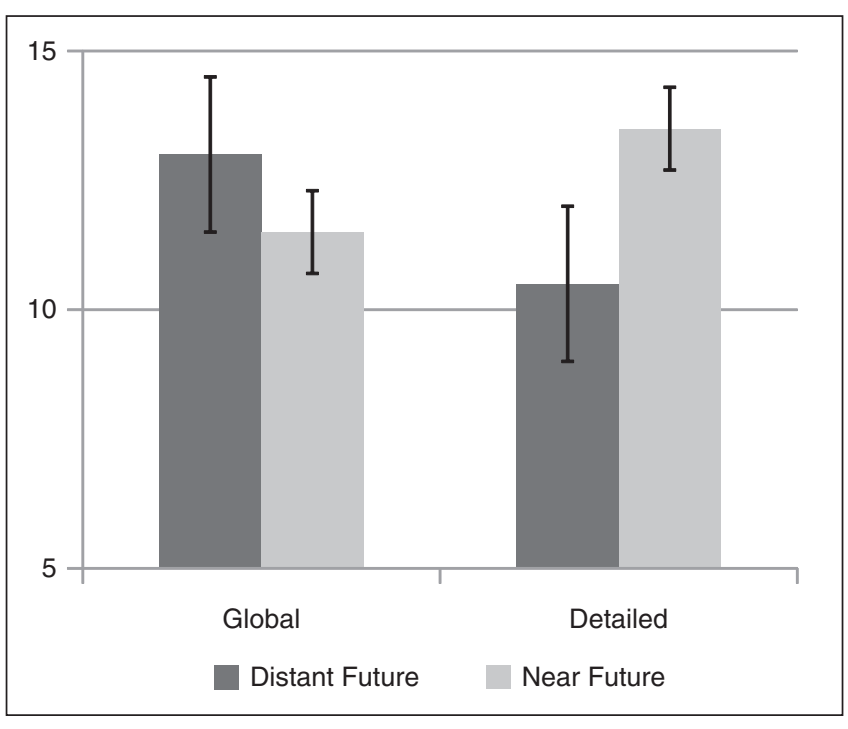

Figure I. Agreement with self-reported thoughts on global versus detailed aspects of an upcoming speech (means with standard errors), as a function of temporal distance

\section{Results and Discussion}

Processing style. The first question of interest is whether the effects of temporal distance were detectable in the processing-style questionnaire. Likert-type responses to each of the five detailed items were summed to provide an overall score for detailed processing, and responses to the global items were summed to provide an overall global processing score. These two measures correlated negatively, $r=-.27$, $p<.05$. The effects of temporal distance on the two measures of processing style were explored in a 2 (temporal distance: near future vs. distant future) $\times 2$ (processing style: global vs. detailed) mixed-model ANOVA, with repeated measures on the second factor. Overall, there was no main effect of temporal distance on processing style scores, $F<1$, and no difference in mean scores on the two subscales, $F<1$. However, the interaction between temporal distance and processing style was significant, $F(1,58)=10.43, M S E=12.89, p<$ $.01, \eta_{p}^{2}=.15$, and is illustrated in Figure 1.This two-way interaction was explored through tests of simple main effects. Participants in the distant-future condition reported reliably greater agreement with the global questions than the detailed questions, $F(1,58)=7.27, M S E=14.68, p<.01, d=0.76$, whereas the reverse was the case for participants in the nearfuture condition, albeit the effect just failed to reach conventional levels of significance on a two-tailed test, $F(1,58)=$ $3.50, p<.07, M S E=11.10, d=0.52$.

Face recognition. The main focus of the study was the impact of temporal distance on subsequent face recognition. A binary logistic regression of lineup accuracy demonstrated reliable differences across condition, $W(2)=9.07, p=.01$, Nagelkerke $R^{2}=.20$, with 8 of $30(26.7 \%)$ participants in the near-future condition correctly identifying the confederate, compared to 14 of $30(46.7 \%)$ control participants and 20 of $30(66.7 \%)$ participants in the distant-future condition.

Relationship between variables. The final analysis concerned the relation between the measures of processing style, as measured by the self-report questionnaire, and performance on the lineup. Neither subscale of the questionnaire correlated significantly with the outcome of the lineup, although both correlations were in the expected directions: The global processing scale correlated positively with lineup accuracy, $r=.25, p<.055$, and the detailed processing scale correlated nonsignificantly but negatively, $r=-.20, p<.12$. Collapsing the two subscales into a single dimension, with global processing scored positively and detailed processing scored negatively, revealed a reliable correlation with identification accuracy, $r=.29, p<.05$.

\section{Summary}

This study shows that orienting people toward a future event has two effects that vary as a function of temporal proximity: Perhaps the least surprising is that people think about the upcoming event differently depending on how close in time it will occur. Thinking about closer events causes people to report thinking more about the features of the event, whereas thinking about an event further away in time causes people to think about general aspects of the event. More surprising is that the same two forms of construal also affect an objective measure of memory: recognition of a photograph of a recently encountered confederate. Those who thought about a closely upcoming event were worse at identifying the confederate than those who thought about an event further in the future, with control performance falling between the two conditions. Thus, the pattern observed using the temporal distance manipulation replicates that seen by Macrae and Lewis (2002), but following a task with no spatial component. In so doing, they demonstrate an effect that Hunt and Carroll (2008) failed to find: an effect of temporal distance on memory performance in a group of participants who had not engaged in a prior verbal description of the target. Notably, our participants were not asked to study the face. Instead they had a naturalistic encounter in the field before they were made aware of the psychological study.

Two alternative possibilities present themselves. First, it is possible that participants in the distant-future condition were induced into a positive mood state through consideration of how they might advance their career or help their university. Conversely, those in the near-future condition might have been anxious because they focused on the details of giving a talk. ${ }^{1}$ Thus, it might be possible that the recognition effects are mediated by mood, either directly because mood alters global-local focus (e.g., Fredrickson \& Branigan, 2005) or indirectly because mood mitigates participants' motivation. We return the role of mood on performance following the presentation of Experiment 2. A second possibility is that 
planning for an event in the near future is more resource demanding than planning for the same event in the distant future. Depleted attentional resources might, then, account for the relatively poor performance displayed by participants in the near-future condition. We address this issue in Experiment 2 , in which our hypotheses specify that under some circumstances memory performance will be improved (rather than impaired) under conditions of low-level construal.

\section{Experiment 2}

Social encounters provide a plethora of information beyond the physical appearance of those we meet. There is ample evidence to suggest that we tend to draw immediate inferences from others' behavior, often resulting in well-elaborated impressions of their personality (Ambady, Hallahan, \& Rosenthal, 1995; Ambady \& Rosenthal, 1992; Winter \& Uleman, 1984). The ways in which people perceive, interpret, and later recall information about others' behavior have received a great deal of empirical attention. In general, evidence suggests that people are best able to retrieve information they have elaborated. A key finding from this literature is that recall of behavioral information is better when it is learned with the goal of forming an impression of a target person than when it is learned with the goal of memorizing it (Hamilton et al., 1980; Wyer et al., 1984). Thus, it seems that a more global processing style benefits not only memory for a person's face but also memory for his or her behavior.

However, an alternative hypothesis deserves attention. Both face-recognition and impression-formation research seem to suggest a general advantage for global (vs. detailed) processing. That is, for both faces and behaviors, one might expect global processing to result in superior performance. However, few studies have investigated the possibility that different types of information might be preferentially recalled under global versus detailed processing conditions. One exception to this rule is suggestive: Research involving individuals with autism spectrum disorder (ASD) reveals that a local (i.e., detailed) processing preference may be responsible for their attention to features rather than configural information when processing faces (see Behrmann, Thomas, \& Humphreys, 2006, for a review). Thus, there is some evidence that detailed processing may actually have an advantage (relative to global processing) when it comes to processing certain types of information. In the case of person memory, one might expect that whereas global processing might benefit recall of abstract information (e.g., a target person's disposition, goals), detailed processing might yield better recall of concrete information (e.g., specific statements made by a target person).

Experiment 2 sought to accomplish two aims. First, we wanted to replicate the results of Experiment 1 using a different set of procedures and a different confederate. Thus, Experiment 2 was carried out in the laboratory and involved an interaction between an experimenter and a female confederate. Second, we aimed to test the two alternative hypotheses discussed previously by determining whether greater psychological distance (and resulting global processing) enhances retrieval of all kinds of information or whether recall of certain types of information might be better under conditions of low psychological distance (and resulting detailed processing). Experiment 2 used a categorization task to obtain an indicator of processing style. Previous research using this task to measure construal level (Liberman, Sagristano, \& Trope, 2002) demonstrated that thinking about the near future leads participants to classify items using a greater number of groups (consistent with a lower level of construal), whereas thinking about the distant future leads them to identify fewer groups (consistent with a higher level of construal).

\section{Method}

Participants. 72 students (51 female) at the University of Plymouth participated in the experiment in partial fulfillment of a course requirement. Participants were tested in groups of 2 to 5 . Data from 16 participants were discarded because, when asked to identify the confederate from the lineup, they reported that they had previously failed to look at her. These participants were evenly distributed across the near-future $(N=8)$ and distant-future $(N=8)$ conditions of the experiment.

Procedure. Upon arriving at the laboratory, participants were greeted by a female experimenter and seated at individual desks, set up such that they each had a clear view of the entrance. When all participants in a session had arrived, the experimenter closed the laboratory door and asked participants to fill out a brief demographic information form. While participants were completing the form, a female confederate abruptly entered the room and announced loudly that she was late for the experiment. After consulting her schedule, the experimenter informed the confederate that she was actually signed up for the following session and so she should come back later. The confederate agreed and left the room. This interaction took place over 1-2 minutes.

Participants were then asked to begin a study on "imagining the future." In this study, they were provided with three scenarios (going camping, moving to a new apartment, going to a car boot sale). Participants in the near-future condition were asked to imagine these scenarios taking place within the next week. Participants in the distantfuture condition were asked to imagine these scenarios taking place the following year. For each scenario, participants were provided with a list of 38 items (adapted from Liberman et al., 2002) and were asked to group the items so that items that "belong together" were in the same group. Participants were allowed up to 3 minutes to form as few or as many groups as they liked. 
After completing the grouping task, participants were introduced to a new study investigating their memory for an event that occurred earlier in the session-namely, the interaction between the confederate and the experimenter. Participants were first presented with a photo lineup and were asked to indicate which of the eight photographs matched the person who had entered the room earlier. As in Experiment 1, a lineup for participants' identifications was developed. The lineup consisted of eight photographs presented simultaneously in a $2 \times 4$ array. Each photograph was approximately $50 \times 50 \mathrm{~mm}$ and showed a frontal view of the head and shoulders of a young woman with light hair, aged 18-30 years. The clothing shown in the lineup photographs differed from that worn by the confederate during the study. The faces were numbered 1-4 (top row) and 5-8 (bottom row). The target's position varied randomly across participants. Participants were required to register a response; that is, there was no "not in lineup" option. Participants were then asked to provide a written description of the event, from the time the confederate entered the room to the time she left. ${ }^{2}$ Finally, participants were asked whether they knew the confederate or had seen her before. None did.

\section{Results and Discussion}

Grouping task. In the present experiment, the total number of groups generated by participants in the near-future and distant-future conditions was analyzed using an independent samples $t$ test. As in previous research, participants in the near-future condition generated significantly more groups $(M=15.00, s=2.70)$ than did those in the distant-future condition $(M=12.25, s=3.26), t(53)=3.40, p=.001, d=$ 0.93 . This difference was also significant for each of the three individual grouping tasks ( $p s \leq .05)$.

\section{Face Recognition}

Participants using a high level of construal (i.e., those in the distant-future condition) were expected to engage in more global processing, which should benefit their ability to recognize faces. To test this, a chi-square test was used to assess the effect of construal level on face-recognition accuracy. Consistent with predictions, participants in the distant-future condition were more likely to correctly identify the confederate $(50 \%)$ than were those in the near-future condition $(14.29 \%), \chi^{2}(1)=8.19, p=.009$.

Narrative descriptions. Participants' descriptions of the event were coded on two dimensions. First, two independent coders (blind to condition) rated each description on the extent to which it included details of the event (e.g., the specific statements made by the confederate or experimenter). Ratings were made on a 1-10 scale with higher numbers reflecting a greater level of detail. Second, the same coders rated each description on the extent to which it referred to the meaning of

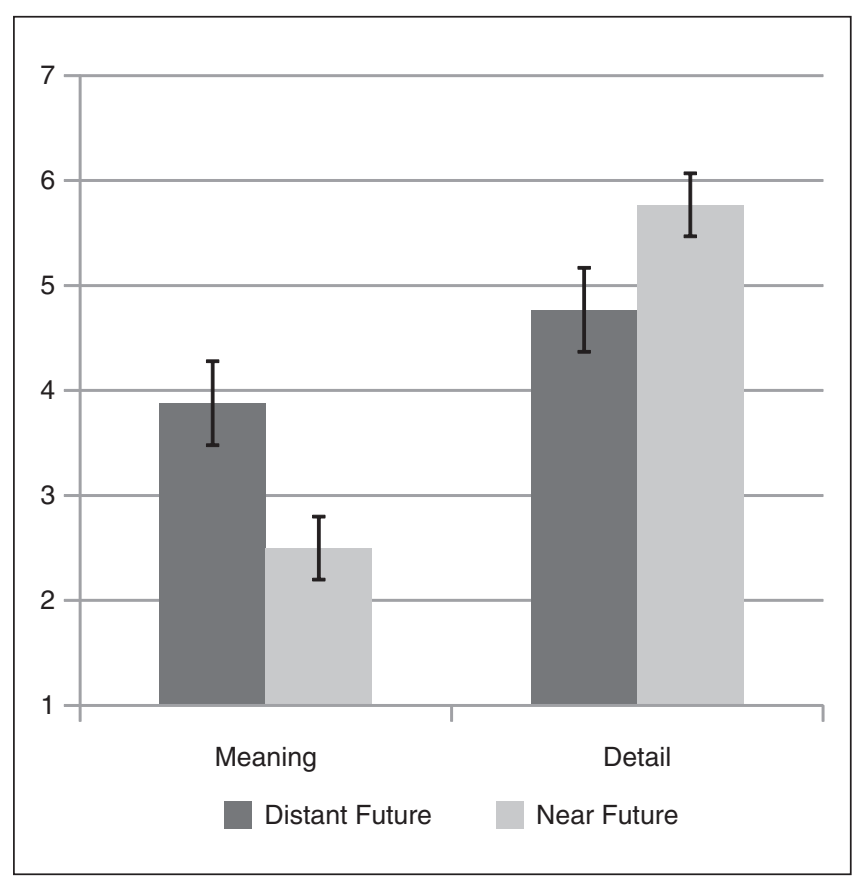

Figure 2. Number of meaning-related versus detail-related items recalled from experimenter-confederate interaction (means with standard errors), as a function of temporal distance

the event (such as the confederate's goals: e.g., "She wanted to do the experiment"; or personality: e.g., "She was a bit rude"). Ratings were made on a 1-10 scale with higher numbers reflecting a greater level of meaning. Interrater reliability was satisfactory for both detail and meaning dimensions $(\alpha \mathrm{s}=.89$ and .92 , respectively), so ratings were averaged for analysis.

Ratings of detail and meaning were first entered as repeated measures in an ANOVA with construal condition entered as a between-subjects factor (see Figure 2). The analysis indicated a significant main effect of rating type, $F(1,54)=$ $42.09, M S E=2.88, p<.001, \eta_{p}^{2}=.44$, such that participants' recall of the event included more detail $(M=5.27, s=1.43)$ than meaning $(M=3.19, s=2.11)$ information. Consistent with predictions, however, this was qualified by a significant interaction between construal and type of description, $F(1$, $54)=13.72, M S E=2.88, p=.001, \eta_{p}^{2}=.20$. To decompose the interaction, simple main effects were computed for each type of description. These revealed that participants in the near-future condition used more details in their descriptions ( $M=5.77, s=1.16)$ than did those in the distant-future condition $(M=4.77, s=1.52), F(1,54)=7.68, M S E=1.82, p=$ $.008, d=0.74$. In contrast, participants in the distant-future condition described a greater level of meaning $(M=3.88, s=$ 2.29) than did those in the near-future condition $(M=2.50$, $s=1.70), F(1,54)=6.52, M S E=4.06, p=.014, d=0.68 .^{3}$

Relationships among variables. Correlational analyses were also carried out to determine the extent to which recollection of the event was related to the number of groups generated during the planning task (which may serve as an indicator of 
global vs. local processing). Ratings of meaning were significantly negatively correlated with the number of groups generated, $r=-.48, p<.001$, whereas ratings of detail were marginally positively correlated with the number of groups generated, $r=.24, p=.07$. Finally, recognition accuracy was marginally negatively correlated with the number of groups generated during the planning task, $r=-.24, p=.08$.

\section{Summary}

Experiment 2 replicated and extended the results obtained in Experiment 1. As in the first study, our manipulation of temporal distance had a number of effects on participants' information processing. First, replicating past research by Liberman et al. (2002), participants who considered events in the near future drew finer distinctions among groups of objects to be used in those events than did those who considered the same events in the distant future. More importantly, replicating Experiment 1, participants who were led to think about the distant future displayed greater accuracy in identifying the confederate encountered earlier, compared to participants led to think about the near future. Finally, although temporal distance had no overall effect on the amount of information recalled from the interaction between the confederate and experimenter, it did significantly affect the type of information recalled. Participants in the distant-future condition were more likely to report information related to the meaning of the interaction (e.g., the confederate's goals) whereas those in the near-future condition were more likely to report specific details from the interaction (e.g., specific statements made by either the confederate or the experimenter).

Thus, Experiment 2 demonstrates that temporal distance influences multiple aspects of perceivers' description of an event during a free recall task. Retrieval of a target's behaviors and dispositions as well as his or her appearance is improved or impaired, depending on a manipulation of psychological distance that took place in an unrelated task, as part of an ostensibly separate experiment. These findings support the hypothesis that construal level has broad implications for how people recall events. As in the first experiment, Experiment 2 showed that alternate indices of processing style derived from the task in which psychological distance was manipulated (in this case, the extent to which participants formed well-differentiated categories) correlate with memory measures in an independent task in a theoretically meaningful way. Category differentiation was positively correlated with recall of event details but negatively correlated with reporting of event meaning.

\section{General Discussion}

The two experiments reported here provide preliminary evidence in support of the hypothesis that psychological distance influences memory for people and events. Collectively, these two studies suggest that temporal distance can influence both recognition memory and recall of a complex event. For face recognition, the same pattern was evident in both studies despite the use of a different confederate, different induction of future focus, and a different lineup. Thus, the effect appears to have some generality.

These findings add to the growing literature showing that performance on face identification tests, particularly those involving lineups, can be impaired, or improved, by prior engagement in different kinds of cognitive task. We have already described the work supporting the transferinappropriate processing account of verbal overshadowing (Schooler \& Engstler-Schooler, 1990; Westerman \& Larsen, 1997) and that demonstrating the effects of prior local or global processing of Navon letters (Macrae \& Lewis, 2002; Perfect et al., 2007). Lewis (2006) showed a similar effect: Participants who engaged in a cryptic crossword subsequently performed more poorly on a lineup task than those who had attempted literal crosswords or sudoku puzzles, or those who had simply read an extract from a novel. More recently Perfect, Weston, Dennis, and Snell (2008) showed a reversed effect using Navon stimuli with reversed precedence to the standard stimuli. By reducing the spatial frequency of the elements (i.e., making the constituent letters farther apart), perceivers' default response shifted to attend to the elements themselves rather than to the global shape they constituted. In that research, participants who were forced to report the global shape subsequently performed more poorly on a lineup, and those who reported the local elements of the shapes subsequently showed improved performance, relative to control.

The present work is consistent with this line of research, in that it shows that identification performance can be enhanced, or impaired, by inducing processing style through temporal distance. Moreover, it builds on the literature in a number of key ways. Most importantly, the studies here show that the effects of processing bias do not simply reduce to a matter of better or worse performance. Processing bias makes memory performance different. It is true that facerecognition performance in a lineup is worse after inducing low-level construal, but aspects of the detail of the event are better recalled. Thus, construal level alters what is retrieved, not how much retrieval takes place. Finally, the studies add to the range of tasks that are shown to produce processing shifts that influence subsequent performance on event memory tasks, and so help constrain theorizing about what the nature of any processing shift might involve.

\section{Construal and Modes of Processing}

The research reported here highlights several intriguing features of construal effects and of transfer-inappropriate processing in general. Previous work on construal effects has often focused on outcomes that relate directly to the manipulation of psychological distance (cf. Förster, Friedman, \& 
Liberman, 2004; Wakslak \& Trope, 2009). For example, considering an event that will take place in the near or distant future affects the way one processes information related to that event (e.g., Liberman et al., 2002). In the current research, temporal distance not only influenced processing of the event being construed but carried over to affect subsequent processing of information (i.e., recognition and recall) in a context that was irrelevant to the task in which high-level versus low-level construal was invoked. Furthermore, our manipulations of temporal distance affected face recognition in ways parallel to more direct manipulations of global versus local processing (e.g., Macrae \& Lewis, 2002; Perfect et al., 2007; Perfect et al., 2008) and affected other aspects of event memory in ways parallel to previous research on processing goals and person memory (e.g., Adaval \& Wyer, 2004; Hamilton et al., 1980).

Thus, our results, in conjunction with previous work on modes of processing and memory, suggest some possible connections between different conceptions of processing style (global vs. local, holistic vs. feature based, high level vs. low level, gestalt vs. detailed, heuristic vs. analytic) that have been discussed in the diverse literatures on face processing and person memory. Although the present experiments were not designed to conclusively determine how these distinctions relate to one another, they do provide potential directions for further investigation. One possibility worth exploring may be that manipulations of temporal distance instigate an attentional bias toward global versus detailed information, which may manifest itself in different ways depending on the information-processing task. When an attentional bias toward global information is induced, cognitive processing about the task at hand (e.g., planning for an event in the distant future) may be oriented toward the abstract meaning of the event (e.g., what goals will be met). Carrying over to a subsequent processing task (e.g., face recognition or recall of an event), this attentional bias may directly affect how new stimuli are processed (e.g., configural information will be used in processing faces), as well as selective memory for meaning-related information. In contrast, when an attentional bias toward detailed information is induced, processing about the task at hand (e.g., planning for an event in the near future) may be oriented toward the details of the event (e.g., the specifics of how the event will unfold). In subsequent tasks, the attentional bias may influence processing of new stimuli (e.g., features will be attended to when processing faces), as well as selective memory for detail-related information.

It is perhaps worth noting that we do not, in these experiments, treat processing style in the primary task (where construal level is invoked) as a "content-free" measure of processing style more generally. Thus, we did not use the questionnaire measure (in Experiment 1) or the categoryformation measure (in Experiment 2) as mediators in our analyses. Although we believe these tasks are strongly influenced by processing style, there are other factors (e.g., expertise with the domains being considered) that are likely to affect participants' responses. In agreement with Wakslak and Trope (2009), we "do not believe that [processing style] in any particular domain should be uniquely responsible for the effects of construal" (p. 57). Thus, although we would expect (as we found) that participants who show a stronger response to construal level in one domain (e.g., planning for a presentation) to would also show a stronger response in subsequent tasks (e.g., recognizing faces), we believe that formal tests of mediation would require process-pure (i.e., content-free and domain-general) measures of processing style.

\section{Alternative Accounts}

The preceding discussion was framed firmly within an information-processing framework, with the current findings explained as a consequence of shifting informationprocessing style. However, an alternative account derives from the "broaden-and-build" theory of emotions (Fredrickson, $1998,2001)$ and the work relating mood, construal level, and face recognition (Johnson \& Fredrickson, 2005). Specifically, the broaden-and-build theory predicts that positive mood states lead people to adopt a broader scope of attention. For instance, Fredrickson and Branigan (2005) demonstrated that participants in a happy mood were more likely to show global response bias toward ambiguous Navon-like stimuli than those in an anxious or neutral mood. More pertinently, Johnson and Fredrickson (2005) explored the effect of manipulating mood state on face-recognition ability. Those in the neutral or anxious-mood conditions showed the wellestablished pattern of superior recognition performance for own-race faces relative to other-race faces. However, those in a happy mood showed equally accurate recognition of otherrace as own-race faces. Thus, there is evidence that positive mood may enhance face recognition, at least for some faces.

However, although we acknowledged the possibility that mood may have played a mediating role in Experiment 1, there are a number of arguments against this interpretation. Foremost is the argument based on parsimony: The informationprocessing account explains the data without the need to impute an unmeasured mood state. The effects of temporal distance resemble those following interventions based on the Navon task (Macrae \& Lewis, 2002), where no mood induction is implied. Furthermore, Experiment 2 showed the same pattern of face-recognition performance despite using an alternate induction of temporal distance. Given that there is no a priori reason to believe that the two temporal distance tasks would result in the same pattern of positive and negative mood across the manipulations of near and far temporal distance, we believe it is more parsimonious to attribute the effects directly to construal, without involving mood in the equation.

Moreover, the evidence that mood influences face recognition is only partially supportive of a mood-based account. 
Johnson and Fredrickson (2005) found that direct manipulations of mood failed to moderate face recognition for ownrace faces and that negative mood failed to influence recognition of other-race faces. For the results of the present studies to be mediated by mood, one would have to argue that our manipulations, involving consideration of future states, resulted in greater mood shifts (and so had a greater impact on face recognition) than having participants watch video clips of standup comedians or horror movies (Johnson \& Fredrickson, 2005). Given that participants in our studies knew that the future orientation tasks were entirely hypothetical, this does not seem plausible.

Finally, it is worth noting that a considerable number of studies have provided more direct evidence against the hypothesis that construal level influences subsequent processing because it affects mood. For instance, Wakslak and Trope (2009) recently reported a series of studies in which construal level (manipulated in various ways) was shown to influence probability judgments. In several of their studies, they also obtained measures of participants' mood following the construal manipulation. In none of these did they find evidence that the effect of construal level on judgments was mediated by mood. Similarly, Förster et al. (2004) reported a series of studies in which the effect of temporal distance on creativity was examined. In establishing that high-level construal produced greater creativity, they ruled out the possibility that the effect was mediated by positive mood. Thus, although we did not obtain direct measures of mood in the current studies, there appears to be reasonable evidence to argue against the possibility that mood played a major role in producing our results.

In addition to the alternative account in terms of mood (detailed previously), a second alternative explanation for the effects of construal on face recognition is that our construal manipulations either enhanced or decreased the attentional resources available to participants. It is plausible to consider that planning for an imminent event might deplete cognitive resources, which might then impair performance on subsequent cognitive tasks (e.g., recognizing faces). However, our finding that cognitive performance is improved on tasks where detailed processing is advantageous (e.g., recalling the details of an event) suggests that it is construal level, and not cognitive resources per se, that is responsible for the range of effects reported here.

\section{Which Memories Are Affected, and When}

Finally, the experiments reported here also open new avenues of research into person memory and event memory in general. Research in the person memory tradition has typically employed experimental contexts that are relatively impoverished, primarily involving the presentation of isolated verbal or pictorial stimuli (cf. Adaval \& Wyer, 2004). In contrast, everyday social encounters involve far more information than behaviors and faces, and are typically context rich. The current research offers an initial investigation into how people process information about these more complex social events. It also raises new questions about how and when processing style may be influential. Everyday events are rich in both social and nonsocial information, much of which can be processed either globally or in a more detailed fashion. For example, the physical environment contains both detailed information (the objects present in a scene) and global or configural information (e.g., where objects are located in relation to each other, what the overall scene looks like). Shifts in processing style that accompany high- versus lowlevel construal may be expected to affect how physical scenes are recalled.

Other questions also require further research to find answers. The experiments reported here imposed a high- or low-level construal after the target event had occurred. Thus, construal level appears to have influenced the retrieval of information that had already been encoded and stored in memory. Indeed, much research on face recognition follows these procedures (Macrae \& Lewis, 2002; Schooler \& Engstler-Schooler, 1990). As such, our results add to existing evidence that different aspects of the very same memory can be retrieved depending on one's current mode of processing.

It remains to be determined what, if any, influence construal level has on the encoding of information. That is, adopting a global versus detailed processing style may be expected to influence attention to and interpretation of information in an event, such that memory storage and not only retrieval is likely to be affected by construal level. For example, when witnessing the same event, individuals operating at a highlevel construal may only attend to (and therefore encode) the meaning of the event, whereas those operating at a low-level construal may be more likely to attend to the details. Similarly, high-level construal is likely to promote attribution and inference processes that would facilitate storage of meaningrelated information, perhaps at the expense of specific details.

\section{An Ecological Caveat}

Strictly speaking, the lineups used in these experiments fail two methodological criteria for appropriate tests of eyewitness memory. The standard approach in eyewitness research is to enable witnesses to reject the entire lineup, as would be the case in the real world where witness may not identify anyone. This is achieved through provision of "unbiased" instructions to the witness that the true perpetrator may or may not be present (Malpass \& Devine, 1981). Such an approach leaves open the possibility that a change in identification could be due to a change in willingness to select anyone, and therefore a methodological approach used in eyewitness research is to include target-absent lineups to 
control for such a response bias. We adopted neither of these procedures, and so it is hard to generalize our findings to real-world conditions. However, the cost of low ecological validity is more than outweighed by the theoretical clarity that our data provide. The lack of unbiased instructions means that there can be no response-bias account of our data, as all participants were required to give an identification response. Our design is effectively a single-trial forced-choice test of memory with a fixed response criterion. Consequently, the observed differences in performance across conditions reflect changes in memory accuracy, not response bias.

\section{Conclusions and Applications}

The research reported here makes a number of novel and important contributions to our understanding of how perceivers recognize others and recall details about their behavior. Few prior studies have investigated memory for both the physical appearance and the behavior of a target person (cf. Adaval \& Wyer, 2004). In this research, we have established that processing style, as determined by construal level, affects multiple aspects of perceivers' recall of an event and the other people involved in it. Construal-level theory provides a useful framework for investigating the influence of processing style on various dimensions of person memory and raises important questions for further research on the factors that influence which information perceivers are able to access from their representation of an event.

Clearly, the results of the present studies raise many questions about how construal might be applied to other situations in which memories of a person or event must be retrieved. While these data are provocative, it will be important for future research to determine the generality and robustness of these effects before we can assess whether they might be used to help witnesses recall past events or recognize previously seen individuals. Many other research questions naturally arise. To what extent can processing style be switched from global to detailed (and back again) to access different aspects of memory? Once high-level or low-level construal is induced, how long-lasting are its effects? How might construal level interact with other factors, such as the group membership of the target being recalled-for example, do temporal distance effects only apply to own-race face recognition? Does temporal distance affect recognition of individual face parts, as well as whole faces from a lineup? We hope that the present research provides a springboard for others to explore these and many other questions.

\section{Acknowledgments}

The authors thank Josie Bannon, Jason Chalangary, Richard Davies, and Naomi Kilgore for their help in the data collection for the present study. They also thank Ian Dennis and Terry Schell for their statistical advice. Finally, they thank Nira Liberman and four other anonymous reviewers for their comments on earlier drafts of this article.

\section{Declaration of Conflicts of Interest}

The authors had no conflicts of interest with respect to the authorship or the publication of this article.

\section{Funding}

The authors received no financial support for the research and/or authorship of this article.

\section{Notes}

1. We are grateful to an anonymous reviewer for this suggestion.

2. It is important to note here that participants were instructed to write what they remembered about the event - in doing so, we assume that they reported those aspects of the event that they were able to retrieve. Because we used a free-recall task rather than a recognition task, we cannot unequivocally rule out the possibility that participants were able to remember more than they reported. However, given the experimental instructions that placed a demand on participants to report what they could, we believe we are safe in concluding that participants reported those aspects of their memories that were spontaneously retrieved in response to the prompt to recall the event.

3. The narrative descriptions were also examined for differences in accuracy and valence. Comparisons on these dimensions are necessarily confounded by differences in the nature of the information recalled. Because near-future participants recalled more specific details of the event, they necessarily recalled more objectively accurate information (in contrast to distantfuture participants who reported more inferences that cannot be classified as objectively correct or incorrect). However, inspection of the objectively verifiable information recalled by participants in each condition indicates that recall accuracy was at ceiling for both near-future $(M=98 \%, s=0.05)$ and distant-future participants $(M=99 \%, s=0.03)$ and did not differ between conditions, $t(54)=0.95, p=.35, d=0.26$. Similarly, because the nature of the staged interaction was mildly negative (the confederate entered and left the room abruptly and had a loud interaction with the experimenter), descriptions of the meaning of the event would necessarily be more negative than reports of the specific details. Hence, because distant-future participants reported more meaning-related information, they also included more negative information in their descriptions. However, inspecting the valence (coded on a 1-10 scale from very negative to very positive) of meaning-related information reported, there was no difference between near-future $(M=4.42, s=$ $0.51)$ and distant-future $(M=4.26, s=0.62)$ participants, $t(35)=$ $0.85, p=.40, d=0.29$.

\section{References}

Adaval, R., \& Wyer, R. S. (2004). Communicating about a social interaction: Effects on memory for protagonists' statements and 
nonverbal behaviors. Journal of Experimental Social Psychology, 40, 450-465.

Ambady, N., Hallahan, M., \& Rosenthal, R. (1995). On judging and being judged accurately in zero-acquaintance situations. Journal of Personality and Social Psychology, 69, 518-529.

Ambady, N., \& Rosenthal, R. (1992). Thin slices of expressive behavior as predictors of interpersonal consequences: A metaanalysis. Psychological Bulletin, 111, 256-274.

Behrmann, M., Thomas, C., \& Humphreys, K. (2006). Seeing it differently: Visual processing in autism. Trends in Cognitive Sciences, 10, 258-264.

Dodson, C. S., Johnson, M. K., \& Schooler, J. W. (1997). The verbal overshadowing effect: Why descriptions impair face recognition. Memory \& Cognition, 25, 129-139.

Förster, J., Friedman, R., \& Liberman, N. (2004). Temporal construal effects on abstract and concrete thinking: Consequences for insight and creative cognition. Journal of Personality and Social Psychology, 87, 177-189.

Fredrickson, B. L. (1998). What good are positive emotions? Review of General Psychology, 2, 300-319.

Fredrickson, B. L. (2001). The role of positive emotions in positive psychology. The broaden-and-build theory of positive emotions. American Psychologist, 56, 218-226.

Fredrickson, B. L., \& Branigan, C. (2005). Positive emotions broaden the scope of attention and thought-action repertoires. Cognition and Emotion, 19, 313-332.

Hamilton, D. L., Katz, L. B., \& Leirer, V. O. (1980). Cognitive representation of personality impressions: Organizational processes in first impression formation. Journal of Personality and Social Psychology, 39, 1050-1063.

Hunt, C., \& Carroll, M. A. (2008). Verbal-overshadowing effect: How temporal perspective may exacerbate or alleviate the processing shift. Applied Cognitive Psychology, 22, 85-94.

Johnson, K. J., \& Fredrickson, B. L. (2005). "We all look the same to me": Positive emotions eliminate the own-race bias in face recognition. Psychological Science, 16, 875-881.

Lewis, M. B. (2006). Eye-witnesses should not do cryptic crosswords prior to identity parades. Perception, 35, 1433-1436.

Liberman, N., \& Förster, J. (2009). Distancing from experienced self: How global versus local perception affects estimation of psychological distance. Journal of Personality and Social Psychology, 97, 203-216.

Liberman, N., Sagristano, M. D., \& Trope, Y. (2002). The effect of temporal distance on level of mental construal. Journal of Experimental Social Psychology, 38, 523-534.

Liberman, N., \& Trope, Y. (2008). The psychology of transcending the here and now. Science, 322, 1201-1205.

Liberman, N., Trope, Y., \& Stephan, E. (2007). Psychological distance. In A. W. Kruglanski \& E. T. Higgins (Eds.), Social psychology: Vol. 2. Handbook of basic principles (pp. 353-383). New York, NY: Guilford.
Macrae, C. N., \& Lewis, H. L. (2002). Do I know you? Processing orientation and face recognition. Psychological Science, 13, 194-196.

Malpass, R. S., \& Devine, P. G. (1981). Eyewitness identification: Lineup instructions and the absence of the offender. Journal of Applied Psychology, 66, 482-489.

Perfect, T. J. (2003). Local processing bias impairs lineup performance. Psychological Reports, 93, 393-394.

Perfect, T. J., Dennis, I., \& Snell, A. (2007). The effects of local and global processing bias on eyewitness identification performance. Memory, 15, 784-798.

Perfect, T. J., Weston, N., Dennis, I., \& Snell, A. (2008). The effects of precedence on Navon-induced processing bias in face recognition. Quarterly Journal of Experimental Psychology, 61, 1479-1486.

Schooler, J. W. (2002). Verbalization produces a transfer inappropriate processing shift. Applied Cognitive Psychology, 16, 989-998.

Schooler, J. W., \& Engstler-Schooler, T. Y. (1990). Verbal overshadowing of visual memories: Some things are better left unsaid. Cognitive Psychology, 22, 36-71.

Trope, Y., \& Liberman, N. (2003). Temporal construal. Psychological Review, 110, 403-421.

Trope, Y., Liberman, N., \& Wakslak, C. (2007). Construal levels and psychological distance: Effects on representation, prediction, evaluation, and behavior. Journal of Consumer Psychology, 17, 83-95.

Wakslak, C., \& Trope, Y. (2009). The effect of construal level on subjective probability judgments, Psychological Science, 20, 52-58.

Westerman, D. L., \& Larsen, J. D. (1997). Verbal overshadowing effect: Evidence for a general shift in processing. American Journal of Psychology, 110, 417-428.

Weston, N. J., \& Perfect, T. J. (2005). The effects of processing bias on the composite effect. Psychonomic Bulletin and Review, 12, 1038-1042.

Weston, N. J., Perfect, T. J., Schooler, J., \& Dennis, I. (2008). Navon processing and verbalization: A holistic/featural distinction. European Journal of Cognitive Psychology, 20, 587-611.

Winter, L., \& Uleman, J. S. (1984). When are social judgments made? Evidence for the spontaneousness of trait inferences. Journal of Personality and Social Psychology, 47, 237-252.

Wyer, R. S., Jr., Bodenhausen, G. V., \& Srull, T. K. (1984). The cognitive representation of persons and groups and its effects on recall and recognition memory. Journal of Experimental Social Psychology, 20, 445-469.

Yin, R. K. (1969). Looking at upside down faces. Journal of Experimental Psychology, 81, 141-145.

Young, A. W., Hellawell, D., \& Hay, D. C. (1987). Configural information in face perception. Perception, 16, 747-759. 